\section{Mortalidade infantil no Brasil e óbitos, na mesma geração, por infarto agudo do miocárdio}

\author{
Infant mortality in Brazil and deaths from acute \\ myocardial infarction in the same generation
}

\author{
1 Instituto Materno Infantil \\ de Pernambuco, \\ Recife, Brasil. \\ Correspondência \\ J. G. B. Alves \\ Instituto Materno Infantil \\ de Pernambuco. \\ Rua dos Coelhos 300 \\ Recife, PE 50070-550, Brasil. \\ joaoguilherme@imip.org.br
}

\begin{abstract}
Low birth weight is a risk factor for cardiovascular diseases, which constitute the main causes of death both in Brazil and worldwide. High infant mortality rates are associated with low birth weight. The aim of this study was to compare mortality from acute myocardial infarction in 2000 in the Northeast and South of Brazil, regions with different infant mortality rates from 1930 to 1950. Mortality from acute myocardial infarction was higher in southern Brazil, with an adjusted coefficient per 100,000 of 60.8 in males and 41.2 in females (South) versus 26.4 in males and 19.2 in females (Northeast). Similar results were found for lung cancer: 22.8 in males and 8.9 in females (South) versus 5.3 in males and 2.8 in females (Northeast). The persistence of different socioeconomic conditions and infant mortality rates between the two regions and the fact that the phenomenon of infant mortality reduction in Brazil has not been translated into important improvements in quality of life impeded an evaluation of the impact of low birth weight on mortality from acute myocardial infarction in this study.
\end{abstract}

Low Birth Weight Infant; Myocardial Infarction; Infant Mortality
João Guilherme Bezerra Alves 1 José Natal Figueiroa 1

\section{Introdução}

O padrão de morbimortalidade no Brasil vem passando por um período de transição nas últimas décadas, com o melhor controle das doenças infecciosas e um predomínio das doenças crônico degenerativas. As doenças cardiovasculares, principal causa de morte no mundo, representaram 27,3\% dos 946.392 óbitos registrados no Brasil no ano de 2000. O infarto agudo do miocárdio causou a morte de 59.287 brasileiros (DATASUS, Informações em Saúde. Estatísticas Vitais - Mortalidade e Nascidos Vivos. http://tabnet.datasus.gov.br/tabnet/tabnet. htm\#EstatVitais, acessado em 09/Mar/2003).

Os fatores de risco conhecidos para as doenças cardiovasculares, como a dislipidemia, o diabetes, a hipertensão arterial, o sedentarismo, a obesidade, não explicam totalmente as diferenças de risco entre indivíduos e populações para a doença cardíaca isquêmica. Recentemente, um novo fator de risco foi identificado, o baixo peso ao nascer, reflexo da subnutrição fetal e no primeiro ano de vida. Em 1977, Forsdahl 1 descreveu uma correlação entre a mortalidade por doença cardiovascular coronariana, entre os anos de 1964-1967, com a taxa de mortalidade infantil na Noruega, no período de 1896 a 1925. Esse autor procurou explicar esses achados sugerindo que o crescimento do indivíduo, realizado em condições sócioeconômicas desfavoráveis e que propiciam uma 
má alimentação em períodos nobres do crescimento, como o período fetal e os primeiros meses de vida, provoca um dano permanente no metabolismo celular e que resulta numa maior vulnerabilidade para o resto da vida.

Diferenças geográficas nas taxas de mortalidade pela doença cardiovascular coronariana, observadas na Inglaterra e no País de Gales, foram relacionadas com diferenças nas taxas de mortalidade infantil setenta anos antes 2 . Em vários outros estudos, o baixo peso ao nascer e a subnutrição no primeiro ano de vida têm se apresentado como um fator de risco para doença cardiovascular coronariana na vida adulta $3,4,5$.

Sendo verdadeira essa correlação entre a subnutrição fetal ou no primeiro ano de vida e maior risco para doença cardiovascular coronariana, é preocupante o impacto que possa provocar nas taxas de mortalidade pelas afecções cardiovasculares nos países em desenvolvimento. Infelizmente, esse tema tem sido alvo de poucas pesquisas nessas regiões. Um estudo, no Sul da Índia, observou maior risco de doença cardiovascular coronariana em homens e mulheres acima de 45 anos, que haviam nascido com baixo peso 6 .

Como as elevadas taxas de mortalidade infantil representam indiretamente o perfil nutricional na vida intra-uterina e no primeiro ano de vida, objetivamos comparar duas regiões do Brasil com diferentes coeficientes de mortalidade infantil entre os anos de 1930 e 1950: média de 185,6 óbitos por mil nascidos vivos no Nordeste e 116 no Sul 7, com as taxas específicas de mortalidade por infarto agudo do miocárdio no ano 2000, ou seja, 50 a 70 anos depois. Pretendeu-se, ainda, verificar se havia diferenças na freqüência de mortalidade precoce por infarto agudo do miocárdio entre as duas regiões. Para maior validação dos dados, também foram utilizadas as taxas de mortalidade por neoplasias malignas da traquéia, brônquios e pulmões, que, reconhecidamente, não guardam relação com o baixo peso ao nascer.

\section{Metodologia}

Tratou-se de um estudo ecológico no qual foram comparadas as taxas específicas de mortalidade por infarto agudo do miocárdio durante o ano de 2000, entre as regiões Nordeste e Sul do Brasil. Os coeficientes de mortalidade por infarto agudo do miocárdio e por câncer foram padronizados por idade e sexo. Utilizou-se o método direto e definiu-se como padrão a população da Região Sul, estratificada por faixa etária e sexo.
Todos os dados foram coletados através do DATASUS (http://tabnet.datasus.gov.br/tabnet/tabnet.htm\#EstatVitais), com exceção dos coeficientes de mortalidade infantil entre os anos de 1930 a 1950, para as regiões Nordeste e Sul do Brasil, que foram obtidos do registro de Simões 7. Para o cálculo das taxas de mortalidade específica por infarto agudo do miocárdio e câncer da traquéia, brônquios e pulmões [(no de mortes/população residente) X 100 mil], foram utilizadas, como populações das regiões Nordeste e Sul, aquelas apontadas no censo demográfico (IBGE: http://www.ibge.gov.br).

Foi empregada a CID-10 8, nos seus capítulos IX (Doenças do Aparelho Circulatório) e II (Neoplasias - tumores; neoplasias malignas da traquéia, brônquios e pulmão), para a determinação das causas específicas do óbito - infarto agudo do miocárdio e câncer. Foi considerada como mortalidade precoce aquela ocorrida antes dos 55 anos de idade no homem e abaixo de 65 anos na mulher.

$\mathrm{Na}$ análise estatística, foi utilizado o teste qui-quadrado de Pearson.

\section{Resultados}

O coeficiente de mortalidade infantil, entre os anos de 1930 a 1950, variou de 193 a 175 (média $=185,6 \pm 1,2)$ no Nordeste e de 121 a 109 (média $=116 \pm 1,1$ ) no Sul 7 . No ano 2000, os coeficientes de mortalidade infantil, no Nordeste e Sul do Brasil, foram de, respectivamente, 53 e 18 mortes por mil nascidos vivos.

Foram registrados 59.287 óbitos por infarto agudo do miocárdio, no Brasil, durante o ano de 2000, sendo 9.794 no Nordeste e 12.771 na Região Sul do país. No mesmo período, houve 14.715 óbitos por neoplasias malignas da traquéia, brônquios e pulmões, sendo 1.713 no Nordeste e 3.966 no Sul. A população residente no Nordeste, em 2000, era de 47.741.711, e no Sul, de 25.107.616 habitantes; o percentual de idosos era de $8,4 \%$ no Nordeste, e, de $9,1 \%$ no Sul.

Os coeficientes de mortalidade por infarto agudo do miocárdio e câncer, ajustados segundo a região e o sexo, encontram-se na Tabela 1. Na Tabela 2, observa-se que os coeficientes de mortalidade por infarto agudo do miocárdio foram mais elevados na Região Sul em todas as faixas etárias, independente do gênero. Os coeficientes de mortalidade por neoplasias malignas da traquéia, brônquios e pulmões foram de 30,9 para 100 mil habitantes no Sul e 3,5 no Nordeste. Na Tabela 3, verifica-se que, em ambos os sexos e em todas as faixas etárias estudadas, os coeficientes de mortalidade por neo- 
Coeficientes de mortalidade por infarto agudo do miocárdio e neoplasias malignas da traquéia, brônquios e pulmões, nas regiões Nordeste e Sul do Brasil, por sexo.

\begin{tabular}{lcccc}
\hline Região & \multicolumn{2}{c}{ Masculino } & \multicolumn{2}{c}{ Sexo } \\
$\begin{array}{l}\text { Infarto agudo } \\
\text { do miocárdio }\end{array}$ & Neoplasia maligna & $\begin{array}{c}\text { Feminino } \\
\text { Infarto agudo } \\
\text { do miocárdio }\end{array}$ & Neoplasia maligna \\
\hline Nordeste* & 26,4 & 5,3 & 19,2 & 2,8 \\
Sul & 60,8 & 22,8 & 41,2 & 8,9 \\
\hline
\end{tabular}

* Coeficiente ajustado, usando como referência a população do Sul, estratificada por idade e sexo.

plasias malignas da traquéia, brônquios e pulmões foram mais elevados na Região Sul.

\section{Discussão}

Vários estudos demonstram que o baixo peso ao nascer está associado com aumento de risco de doença cardiovascular coronariana, assim como o ganho ponderal insuficiente no primeiro ano de vida e o rápido ganho de peso após os seis anos de idade 3,9,10. Admite-se que isso seja uma conseqüência de alterações fisiológicas e metabólicas persistentes que acompanham o crescimento fetal insatisfatório; o feto se adapta à subnutrição na vida intra-uterina modificando o seu metabolismo, alterando a sua produção hormonal e a sensibilidade dos tecidos, redistribuindo o fluxo sanguíneo e diminuindo a taxa de crescimento. Adaptações à subnutrição, que ocorrem durante os primórdios do desenvolvimento infantil, alteram permanentemente a estrutura e função do corpo. O que antes era considerado como uma hipótese (Hipótese de Barker), hoje, é aceito como verdade, ou seja, a subnutrição fetal ou no primeiro ano de vida, principalmente quando seguida de plena recuperação nutricional ou até excesso de peso ou obesidade, representa um fator de risco para o desenvolvimento de doença cardiovascular coronariana 11.

Como não tínhamos o dado de peso ao nascimento das populações estudadas, utilizamos uma medida indireta, representada pelo coeficiente de mortalidade infantil. Elevadas taxas de mortalidade estão associadas com altos índices de baixo peso ao nascer e de subnutrição no primeiro ano de vida. Vários outros estudos também utilizaram os coeficientes de mortalidade infantil como medida indireta do baixo peso ao nascer 1,2 . São incontestáveis as diferenças de taxas de mortalidade infantil entre as
Tabela 2

Coeficientes de mortalidade por infarto agudo do miocárdio nas regiões Nordeste e Sul do Brasil, por sexo e faixa etária.

\begin{tabular}{lrrrr}
\hline \multirow{2}{*}{ Faixa etária (anos) } & \multicolumn{2}{c}{ Nordeste } & \multicolumn{2}{c}{ Sul } \\
& Masculino & Feminino & Masculino & Feminino \\
\hline$>75$ & 310,4 & 242,1 & 876,2 & 682,3 \\
$65-74$ & 193,4 & 118,8 & 463,2 & 249,0 \\
$55-64$ & 100,8 & 55,3 & 227,3 & 105,7 \\
$45-54$ & 45,6 & 24,8 & 90,0 & 35,9 \\
$35-44$ & 14,5 & 7,9 & 25,9 & 10,6 \\
$<34$ & 1,0 & 0,4 & 1,4 & 0,6 \\
\hline
\end{tabular}

Tabela 3

Coeficientes de mortalidade por neoplasias malignas da traquéia, brônquios e pulmões, nas regiões Nordeste e Sul do Brasil, por sexo e faixa etária.

\begin{tabular}{lrrrr}
\hline \multirow{2}{*}{ Faixa etária (anos) } & \multicolumn{2}{c}{ Nordeste } & \multicolumn{2}{c}{ Sul } \\
& Masculino & Feminino & Masculino & Feminino \\
\hline$>75$ & 50,6 & 18,4 & 279,9 & 81,0 \\
$65-74$ & 44,9 & 17,1 & 210,9 & 66,8 \\
$55-64$ & 25,2 & 11,7 & 101,3 & 34,5 \\
$45-54$ & 8,0 & 5,6 & 29,0 & 9,7 \\
$35-44$ & 1,3 & 1,2 & 4,5 & 3,0 \\
$<34$ & 0,2 & 0,1 & 0,1 & 0,2 \\
\hline
\end{tabular}


regiões Sul e Nordeste do Brasil durante o período estudado (1930/2000). Na Região Nordeste, por exemplo, somente ao final da década de 40 , começa a se observar tendências na redução da mortalidade infantil, mesmo assim, num ritmo bem inferior ao da Região Sul 7 .

O fato de não termos observado coeficientes de mortalidade por infarto agudo do miocárdio mais elevados na Região Nordeste, onde a mortalidade infantil era mais alta há 50-70 anos do que na Região Sul, talvez possa ser justificado por alguns motivos. Inicialmente, no Brasil, a situação da queda da mortalidade infantil ocorreu de forma distinta da observada em outros países estudados, onde a diminuição dos coeficientes de mortalidade infantil foi acompanhada de importante melhoria na qualidade de vida das pessoas, inclusive com fortes implicações nas mudanças de hábitos alimentares, propiciando aumento nas taxas de excesso de peso e obesidade. No Brasil, a diminuição das taxas de mortalidade infantil registradas nas últimas décadas deveu-se a vários fenômenos, como: diminuição da taxa de fecundidade total e melhoria do nível de instrução materna, aumento da oferta de serviços sanitários e da cobertura da assistência ambulatorial e hospitalar, desenvolvimento de programas específicos voltados à atenção da saúde da mulher e da criança 12 . Não foram registradas significativas melhorias das condições de vida da população brasileira, diferentemente do que ocorreu em outros países, onde as pesquisas, com metodologia semelhante, apontaram uma forte associação da mortalidade infantil elevada com letalidade por infarto agudo do miocárdio na vida adulta 1,2 .

Acreditamos que o fenômeno da migração populacional, mais observado, nessa época, das regiões Nordeste para Sudeste do que para o Sul, pouco tenha influenciado esses resultados. A possibilidade do sub-registro não pode deixar de ser mencionada, em especial na região menos desenvolvida, o Nordeste, e principalmente para os coeficientes de mortalidade infantil, aqui estudados entre os anos 1930/1950. Entretanto, como o efeito do sub-registro seria o de subdimensionar as taxas de mortalidade infantil na Região Nordeste, e trabalhamos embasados na idéia de comparar regiões com distintas taxas de mortalidade infantil, isso só faria aumentar a diferença entre os coeficientes das regiões Nordeste e Sul, o que contribuiria para testar a hipótese do estudo.

Em relação aos coeficientes de infarto agudo do miocárdio e de câncer, obtidos através do DATASUS e relativos ao ano 2000, a possibilidade de sub-registro é bem menor. Entretan- to, em relação à qualidade desses dados, registre-se que $14,3 \%$ do total de óbitos, nesse período, foram por causas mal definidas 13 , variando de $27,5 \%$ na Região Nordeste e de $6,2 \%$ no Sul do país. As causas mal definidas têm se mantido entre as cinco primeiras causas de óbito no Brasil (DATASUS: http://tabnet.datasus. gov.br/tabnet/tabnet.htm\#EstatVitais), o que prejudica a análise da mortalidade. Parte dos óbitos por infarto agudo do miocárdio pode estar registrada como por causas mal definidas 14 .

Observamos ainda que, no ano 2000, os coeficientes de mortalidade infantil permaneceram mais elevados no Nordeste, quase três vezes maior, ou seja, proporcionalmente mais elevados do que entre os anos 1930/1950, quando a mortalidade infantil era $62,9 \%$ mais alta no Nordeste. Isso implica que, nessa região, as condições que propiciam uma retomada do crescimento pondo-estatural, seja na infância ou adolescência, e que concorrem para o desenvolvimento das alterações etiopatogênicas da aterosclerose, segundo os estudos de Barker e outros 5,9,11, tenham ocorrido com menor intensidade. Por outro lado, essas condições facilitadoras ocorreram com maior freqüência na Região Sul, o que talvez possa justificar, em parte, a maior taxa de letalidade pelo infarto agudo do miocárdio.

O fato de termos observado taxas de mortalidade precoce por infarto agudo do miocárdio e câncer de pulmão mais elevadas na Região Nordeste pode ser justificado pelas diferenças nas condições sócio-econômicas, entre as duas regiões, existentes nesses últimos 50-70 anos. Condições de pobreza na vida adulta são causas conhecidas de aumento do risco de morte por doença coronariana, porém se esses efeitos interagem com aqueles do crescimento fetal ou na infância, ainda não são bem estudados 15. A melhor condição no Sul favorece diagnósticos e terapêuticas mais precoces e um conseqüente melhor prognóstico. Sendo assim, isso poderia justificar o achado de uma mortalidade precoce por neoplasias malignas da traquéia, brônquios e pulmões, na faixa etária inferior aos 34 anos, mais elevada no Nordeste. Há ainda o registro de que as taxas de mortalidade por doenças infecciosas e parasitárias ainda são elevadas no Nordeste do Brasil, o que indiretamente indica condições sanitárias desfavoráveis.

Acreditamos que, com a metodologia empregada, haja vista a queda das taxas de mortalidade infantil observada no Brasil não refletir necessariamente melhores condições de vida e ainda se manter díspar entre as duas regiões estudadas, não tenha sido possível observar os 
efeitos da subnutrição fetal ou no primeiro ano de vida sobre a letalidade por doença cardiovascular coronariana na vida adulta. Pela dimensão epidemiológica que esse tema representa, especialmente para os países em desenvolvimento que enfrentam um momento de transição epidemiológica com elevados índices de baixo peso ao nascer e desnutrição no primeiro ano de vida, acompanhado de elevação das taxas de excesso de peso e obesidade, um maior número de estudos se faz necessário com a verificação das taxas de mortalidade por doença cardiovascular coronariana em pessoas que tiveram baixo peso ao nascer.

\section{Resumo}

Baixo peso ao nascer, fator de risco recentemente descrito para as doenças cardiovasculares, está associado com mortalidade infantil elevada. Foram comparadas as taxas de mortalidade por infarto agudo do miocárdio no ano 2000, registradas nas regiões Nordeste e Sul do Brasil, com os coeficientes de mortalidade infantil entre os anos 1930/1950. Entre os anos 1930/1950, o Nordeste apresentava um coeficiente médio de mortalidade infantil de 185 por mil nascidos vivos, e a Região Sul, 116 por mil nascidos vivos. Observou-se uma maior mortalidade por infarto agudo do miocárdio na Região Sul (coeficientes ajustados de 60,8 e 41,2 vs. 26,4 e 19,2 por 100 mil habitantes, respectivamente para o sexo masculino e feminino). A desigualdade entre as taxas de mortalidade infantil no Nordeste e Sul no período estudado, ao lado de que o fenômeno da redução da mortalidade infantil não ter representado melhorias importantes das condições de vida, impediu a avaliação do impacto do baixo peso ao nascer sobre as taxas de mortalidade por infarto agudo do miocárdio na vida adulta.

Recém-Nascido de Baixo Peso; Infarto do Miocárdio; Mortalidade Infantil

\section{Colaboradores}

J. G. B. Alves elaborou o artigo participando de todas as fases da pesquisa. J. N. Figueiroa colaborou na modelagem estatística dos resultados e na redação da discussão. 


\section{Referências}

1. Forsdahl A. Are poor living conditions in childhood and adolescence an important risk factor for arteriosclerotic disease? Br J Prev Soc Med 1977; 31:91-5.

2. Barker DJ, Osmond C. Infant mortality, childhood nutrition, and ischaemic heart disease in England and Wales. Lancet 1986; 1:1077-81.

3. Barker DJ, Winter PD, Osmond C, Margetts B. Weight in infancy and death from ischaemic heart disease. Lancet 1989; 2:577-80.

4. Rich-Edwards JW, Stampfer MJ, Mansin J, Rosner B, Hankinson SE, Colditz GA, et al. Birthweight and risk of cardiovascular disease in a cohort of women followed up since. BMJ 1997; 315:396-400.

5. Frankel S, Elwood P, Sweetnam P, Yarnell J, Davey SG. Birthweight, body mass index and incident coronary heart disease. Lancet 1996; 348:1478-80.

6. Stein C, Fall CHD, Kumaran K, Osmond C, Cox V, Barker DJP. Fetal growth and coronary heart disease in South India. Lancet 1996; 348:1269-73.

7. Simões CC. Estimativas da mortalidade infantil por microrregiões e municípios. Brasília: Ministério da Saúde; 1999.

8. Organização Mundial da Saúde. Classificação estatística internacional de doenças e problemas relacionados à saúde, 10a Revisão. São Paulo: Centro Colaborador da OMS para a Classificação de Doenças em Português; 1995.
9. Leon DA, Lithell HO, Vagero D, Koupilova I, Mohsen R, Berglund L, et al. Reduced fetal growth rate and increased risk of death from ischaemic heart disease: cohort study of 15,000 Swedish men and women born 1915-29. BMJ 1998; 317:241-5.

10. Eriksson JG, Forsén T, Tuomilehto O, Osmond C. Early growth and coronary heart disease in later life: longitudinal study. BMJ 2001; 322:949-53.

11. Barker DJP. Fetal origins of coronary heart disease. BMJ 1995; 311:171-4.

12. Frias PG, Vanderlei LC, Vidal S, Leal MC. Morbidade e mortalidade infantil e na infância. In: Alves JGB, Ferreira OS, Maggi RS, organizadores. Fernando Figueira - Pediatria - IMIP. 3a Ed. Porto Alegre: Medsi; 2004. p. 47-59.

13. Laurenti R. Mortalidade Brasil - 2000. http://tabnet.datasus.gov.br/cgi/sim/dados/cid10/docs/es tudo2000.pdf (acessado em 27/Mai/2004).

14. Oliveira GMM, Klein CH, Silva NAS. Análise crítica das mudanças das taxas de mortalidade por doenças do aparelho circulatório ocorridas entre 1980 e 2000, no Estado do Rio de Janeiro. http:// www.socerj.org.br/revista/abr 2003/art01.pdf (acessado em 27/Mai/2004).

15. Acheson D. Independent inquiry into inequalities in health. London: Her Majesty's Stationery Office; 1998.

Recebido em 22/Out/2003

Versão final reapresentada em 18/Jun/2004

Aprovado em 09/Jul/2004 